\title{
Searches for heavy resonances at the LHC
}

\author{
Anirban Saha*t \\ INFN Sezione di Perugia, Università di Perugia, Perugia, Italy \\ E-mail: saha.anirban@pg.infn. it
}

Heavy resonances decaying into a pair of particles is an obvious place to look for phenomena beyond the Standard Model (SM). Results from searches for new resonances in the final states like pairs of leptons, leptons + missing transverse energy, photon + jet, and lepton + jets are summarized in this proceeding. Various models for heavy resonances are considered such as Z', W', Randall-Sundrum Gravitons, large extra dimension scenario, excited quarks. All these searches are performed on the data collected with the ATLAS and CMS experiments in protonproton collisions at the LHC at the center-of-mass energy of $8 \mathrm{TeV}$.

XXII International Workshop on Deep Inelastic Scattering and Related Subjects - DIS 2014 April 28 - May 2, 2013

Warsaw, Poland

\footnotetext{
* Speaker.

†n behalf of the ATLAS and CMS collaborations.
} 


\section{Introduction}

The Standard Model (SM) of particle physics has so far been very successful theory. But it can not explain many of the questions like the constituents of Dark Matter (DM), the hierarchy problem etc., which suggests that the SM may be an effective, low-energy approximation of a more fundamental theory. Many Beyond the Standard Model (BSM) theories predict heavier gauge boson. An extensive effort of searching for those heavy resonances in many BSM scenarios are done by both the ATLAS [1] and the CMS [2] collaborations in proton-proton collisions at the Large Hadron Collider (LHC) at the center of mass energy of $8 \mathrm{TeV}$. This proceeding summarizes the results for the search for the heavy resonances the data collected in the Run-I of the LHC.

\section{Searches for high mas resonances in di-lepton channels}

Many theories beyond the Standard Model (SM) predict a heavy neutral spin-1 particle (Z'), which then decays to a pair of leptons. The most simple model is the Sequential Standard Model (SSM), where new gauge boson only differ from the SM Z boson by the mass. There are other model theoretically motivated by Grand Unified Theories (GUT), where the additional neutral gauge boson arises. The distribution of the invariant mass of two muons in the final state is shown in figure 1. As no significant excess is observed and limits are set at the 95\% confidence level as shown in 2 for both the experiments. Different conventions are followed while setting cross-section limits, ATLAS uses the shape obtained from a Z', which does not interfere with the SM Z boson, while CMS models the signal as having zero intrinstic width. Both the experiments exclude the benchmark $Z_{\mathrm{SSM}}^{\prime}$ for masses below $2.9 \mathrm{TeV}$ [3], [4].
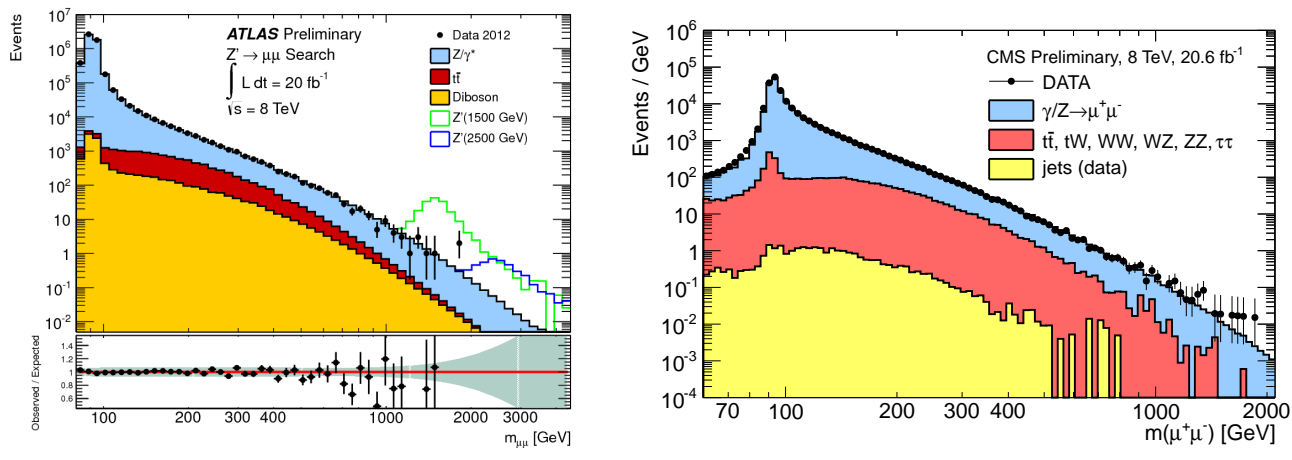

Figure 1: The invariant mass spectrum for two muons for ATLAS (left) and CMS (right) experiments.

\section{Searches for high mass resonances in lepton and $E_{T}$ final states}

The other important final state to look for new resonances is the lepton and $\mathbb{E}_{T}$ final states, where the events are collected with single lepton trigger and selected with lepton identification criteria to reduce the fake rate as much as possible. The distribution of the observed transverse mass spectra of electron and $\mathbb{E}_{T}$ final state are shown in figure 3 along with expected backgrounds for both the experiments. No significant excess is observed and limits are set at the $95 \%$ confidence 

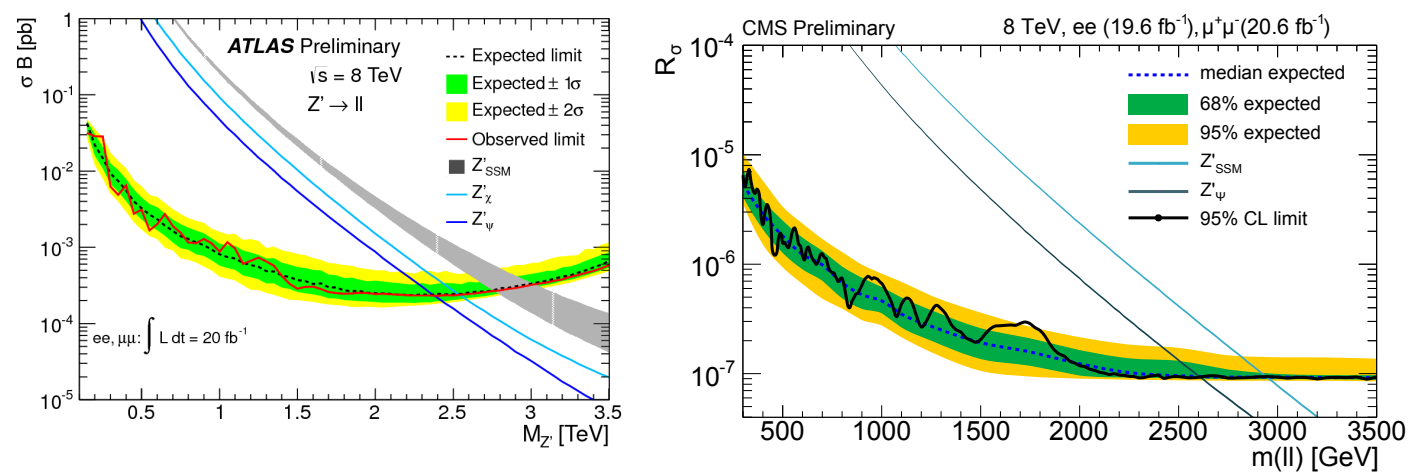

Figure 2: $95 \%$ confidence level limit on $\sigma \times \mathrm{BR}$ for the ATLAS (left) and on $R_{\sigma}$ for the CMS (right) experiment, where $R_{\sigma}$ is the ratio of the inclusive cross-section of the processes $Z^{\prime} \rightarrow l l$ to $Z \rightarrow l l$.

level as shown in figure 4. ATLAS and CMS experiments exclude the benchmark $W_{\mathrm{SSM}}^{\prime}$ for masses below $3.27 \mathrm{TeV}$ and $3.35 \mathrm{TeV}$ [5], [6] respectively.
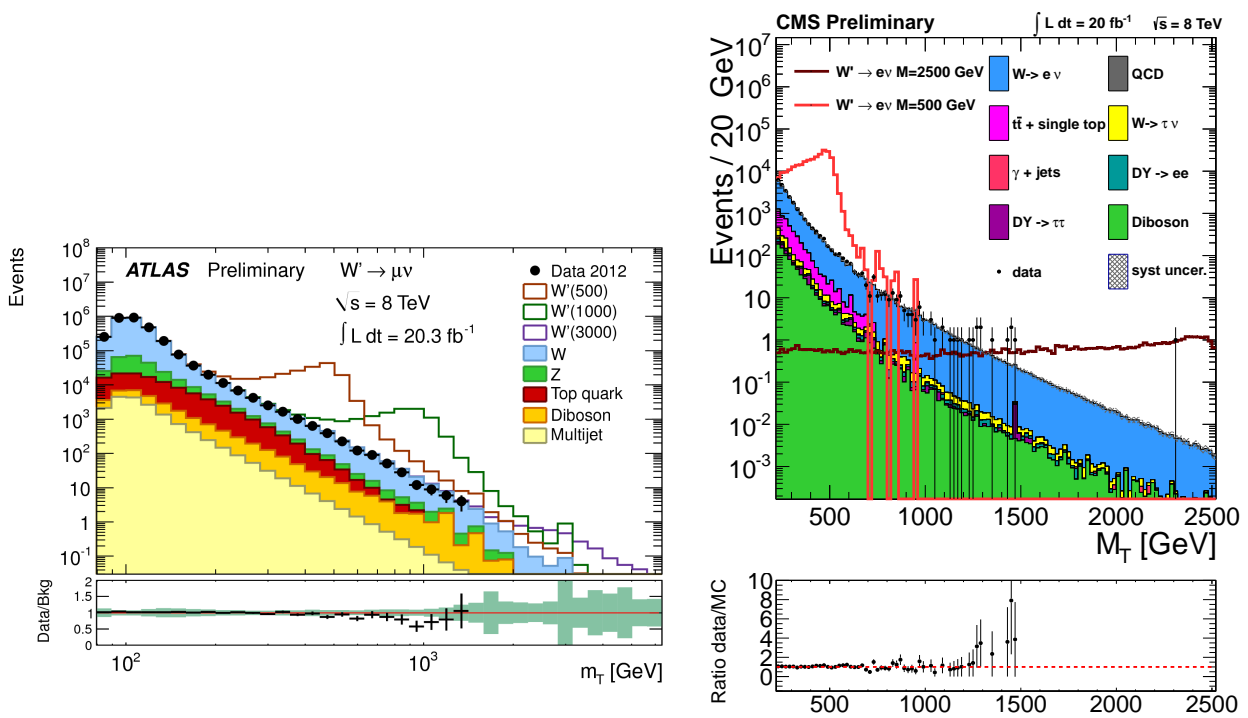

Figure 3: The transverse mass spectrum for electrons and $\mathbb{E}_{T}$ final states for ATLAS (left) and CMS (right) experiments.

\section{Searches for high mass resonances in photon and jet final states}

Many extensions of the SM predict that quarks are composite objects. The most compelling signature for the substructure of quarks would be the existence of the excited state of quark $\left(q^{*}\right)$, which couples with the ordinary quarks, gluons and leptons via the contact interactions. In protonproton collisions excited quarks are predominantly produced by the quark-gluon annihilation process, the excited quarks then decay into a quark and a gauge boson $(\gamma, \mathrm{g}, \mathrm{W}, \mathrm{Z})$. The search for a resonance in the $\gamma+$ jet final state is done by both the ATLAS and CMS collaborations. After the event selection criteria, the final $\gamma+$ jet mass distribution from the data is compared with the 

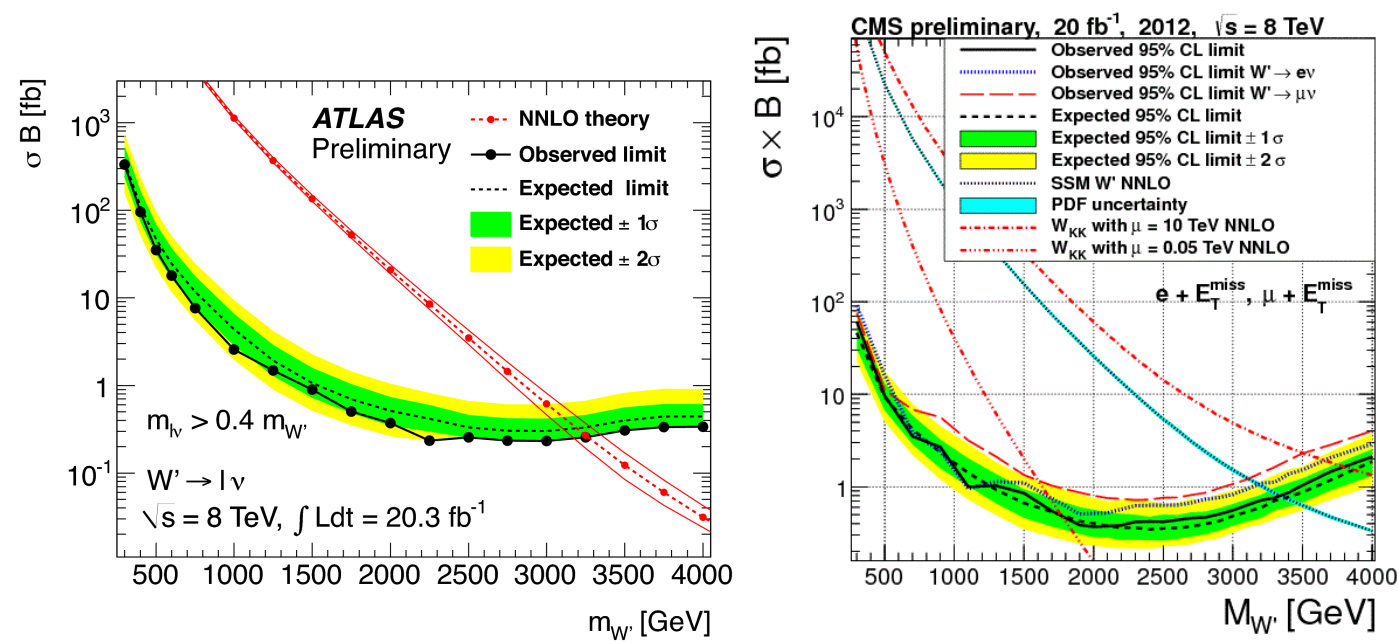

Figure 4: $95 \%$ confidence level limit on $\sigma \times$ BR for the ATLAS (left) and the CMS (right) experiment.

expected monte-carlo prediction, and the comparison is shown in figure 5, along with three different mass of the excited quarks. Since no significant excess in data is observed, 95\% confidence level limits are set on the production cross-section as a function of excited quark mass. The figure 6 shows the observed and expected limit as a function of the mass of the excited quarks. Both the experiments exclude the excited quark mass below $3.5 \mathrm{TeV}$ [7], [8] at 95\% confidence level.
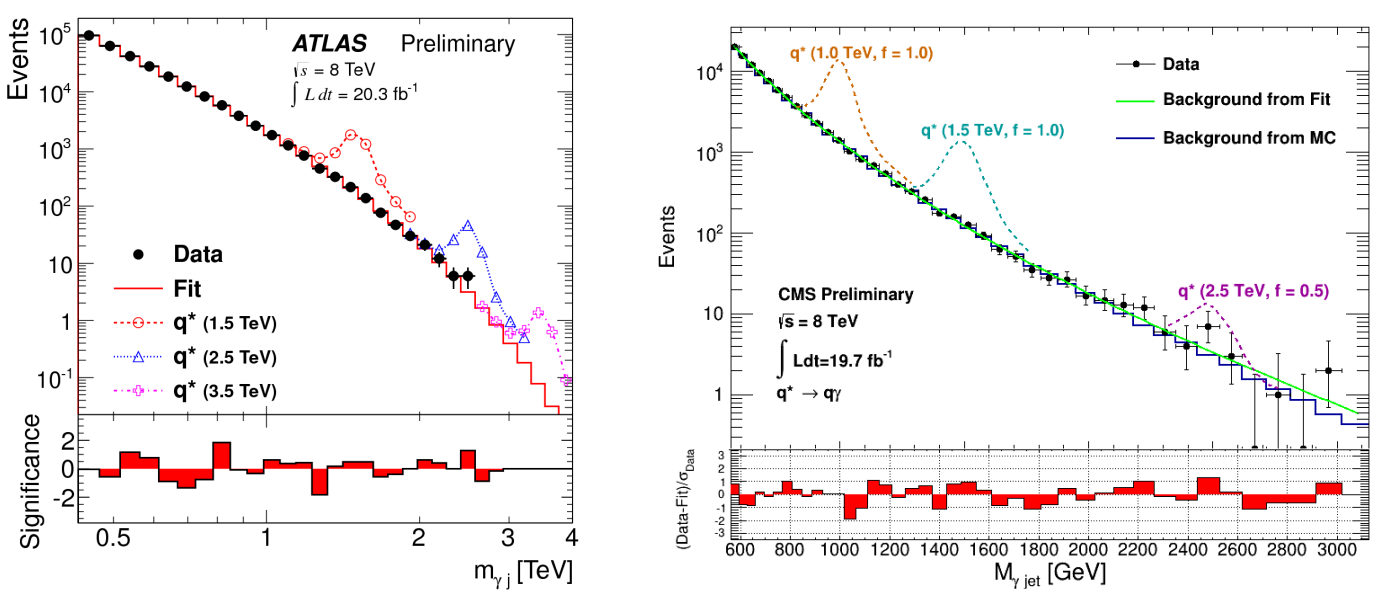

Figure 5: The invariant mass spectrum for $\gamma+$ jets final states for ATLAS (left) and CMS (right) experiments.

\section{Searches for scalar leptoquarks}

To explain the underlying relationship between the leptons and the quarks, many extensions of the SM, like grand unified theories, composite models, superstring-inspired models, predict the existence of particles that can couple to both quarks and leptons. The dominant pair-production mechanisms for leptoquarks at the LHC are gluon-gluon fusion and quark-antiquark annihilation, and the cross sections for these processes depend only on the leptoquark mass and spin. In this 

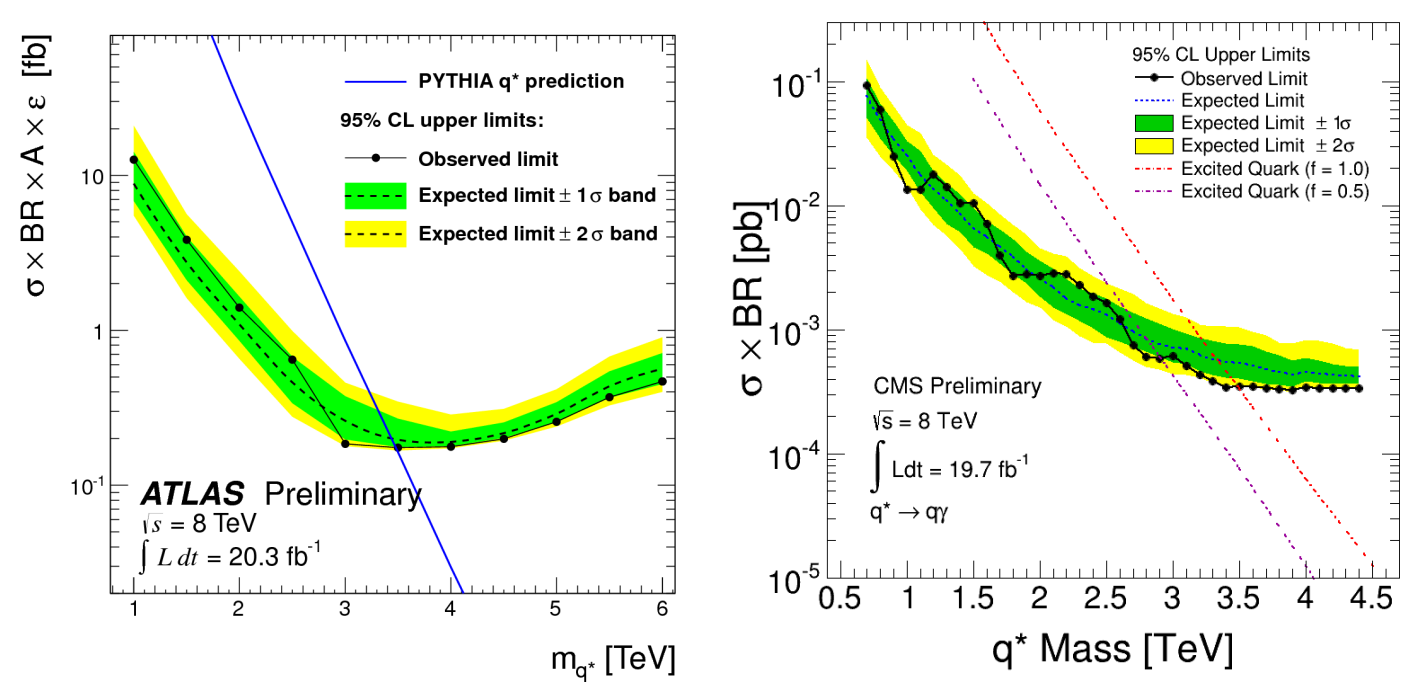

Figure 6: $95 \%$ confidence level limit on $\sigma \times$ BR for the ATLAS (left) and the CMS (right) experiment.

proceeding, a search for third-generation scalar leptoquarks, each decaying to a tau lepton and $\mathrm{a} b$ or top quark, is presented. We assume $100 \%$ breaching fraction for the leptoquark decay to a pair of $\tau$ lepton and $\mathrm{b} /$ top quark. The $\mathrm{S}_{\mathrm{T}}$ distribution after the applying the event selection criteria is used to extract limits on the leptoquark mass, where $S_{T}$ is defined as the scalar sum of the transverse momentum of all the final state particles. The final $\mathrm{S}_{\mathrm{T}}$ distribution for the third generation leptoquark search is shown in figure 7. As the data agrees well with the SM background prediction, a limit is set on the product of the cross section for pair production of third-generation leptoquarks and the square of the branching fraction (B), for the leptoquark decaying to $\tau+b, \tau+$ top final states. The final limit plots are shown in figure 8 . The third generation leptoquark masses below $740 \mathrm{GeV}$ and $550 \mathrm{GeV}$ are excluded from the $\tau+\mathrm{b}$ and $\tau+$ top final states respectively [9], [10].

\section{Summary}

In this proceeding the search results for heavy resonances in the di-lepton, lepton $+\mathbb{E}_{T}, \gamma+$ jets and $\tau+$ jets final states are presented. No significant excess is observed in any of the channels and and limits at the $95 \%$ confidence level have been set on a variety of models. We are looking forward for the start-up of the LHC in 2015 for exciting new results at center-of-mass energy of 13 $\mathrm{TeV}$.

\section{References}

[1] The ATLAS collaboration, 2008 JINST 3 S08003.

[2] The CMS collaboration, 2008 JINST 3 S08004.

[3] The ATLAS collaboration, ATLAS-CONF-2013-017.

[4] The CMS collaboration, CMS-PAS-EXO-12-061. 

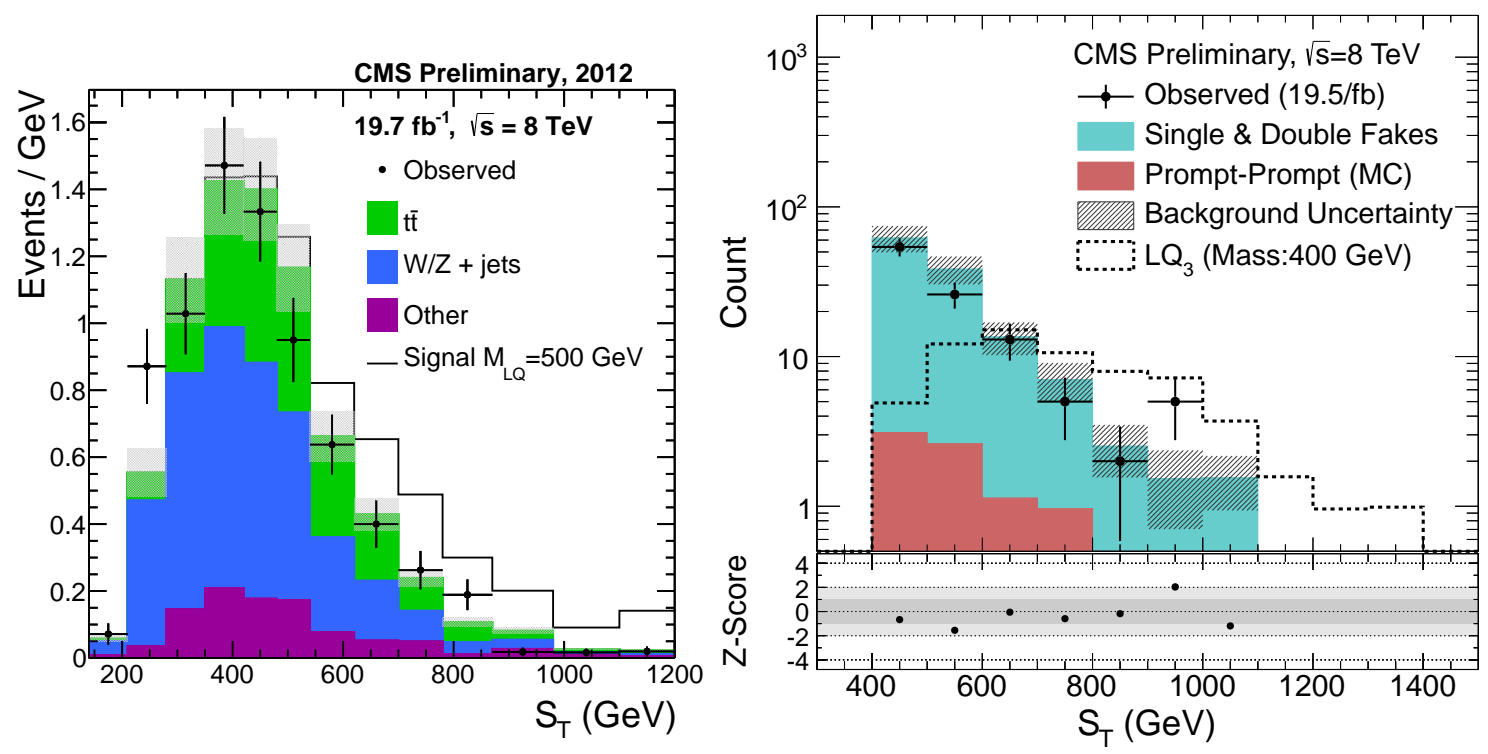

Figure 7: Final $\mathrm{S}_{\mathrm{T}}$ distribution for the third generation leptoquark search in $\tau+\mathrm{b}$ (left) and $\tau+$ top (right) final states.
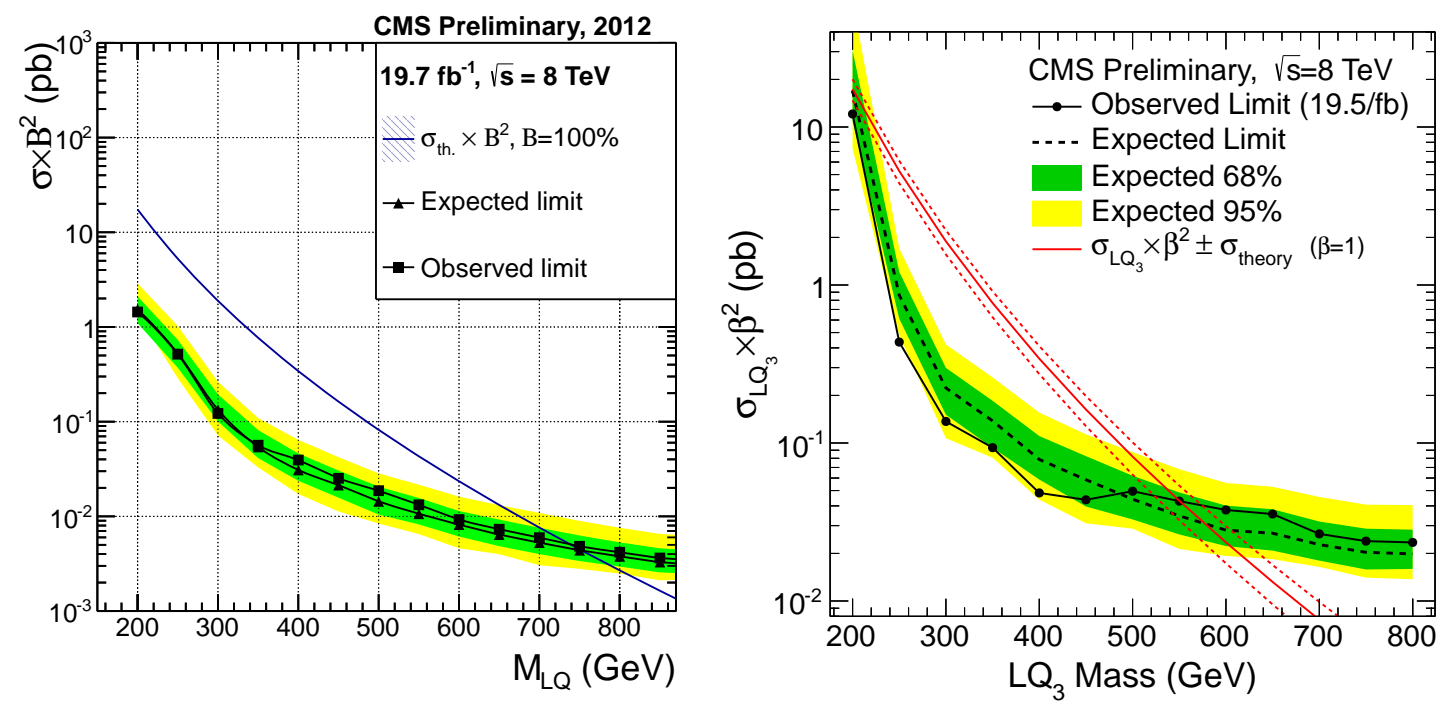

Figure 8: The expected and observed upper limit on $\sigma \times \mathrm{B}^{2}$ at the $95 \% \mathrm{CL}$, as a function of third generation leptoquark mass, for $\tau+\mathrm{b}$ (left) and $\tau+$ top (right) final states, assuming $100 \%$ breaching fraction for the leptoquark decay to a pair of $\tau$ lepton and b/top quark.

[5] The ATLAS collaboration, ATLAS-CONF-2014-017.

[6] The CMS collaboration, CMS-PAS-EXO-12-060.

[7] The ATLAS collaboration, PLB 728, 562 (2013).

[8] The CMS collaboration, CMS-PAS-EXO-13-003.

[9] The CMS collaboration, CMS-PAS-EXO-12-032.

[10] The CMS collaboration, CMS-PAS-EXO-12-030. 